\title{
Cardioprotective Effect of Olive Oil Against Ischemia Reperfusion-induced Cardiac Arrhythmia in Isolated Diabetic Rat Heart
}

\author{
Ishfaq A. Bukhari ${ }^{1}$, Osama Y. Mohamed ${ }^{1}$, Abdulrahman A. Almotrefi ${ }^{1}$, Bassem Y. Sheikh ${ }^{2}$, Omnia Nayel $^{3}$ \\ , Fahim Vohra ${ }^{4}$, Sibtain Afzal ${ }^{5}$ \\ 1. Pharmacology, College of Medicine, King Saud University, Riyadh, SAU 2. Neurosurgery, College of Medicine, Taibah \\ University, Almadinah Almunawara, Madinah, SAU 3. Pharmacology, College of Medicine, University of Alexandria, \\ Alexandria, EGY 4. Prosthetic Dental Sciences, College of Dentistry, King Saud University, Riyadh, SAU 5. Allergy and \\ Immunology, College of Medicine, King Saud University, Riyadh, SAU
}

Corresponding author: Ishfaq A. Bukhari, bukharirph@yahoo.com

\section{Abstract}

\section{Background}

Olive oil is rich in monounsaturated fatty acids and has been reported for a variety of beneficial cardiovascular effects, including blood pressure lowering, anti-platelet, anti-diabetic, and antiinflammatory effects. Diabetes is a major risk factor for cardiac dysfunctions, and olive oil prevents diabetes-induced adverse myocardial remodeling.

\section{Objective}

The study aimed to evaluate the effects of olive oil against streptozotocin-induced cardiac dysfunction in animal models of diabetes and ischemia and reperfusion (I/R)-induced cardiac arrhythmias.

\section{Methods}

Diabetes was induced in male rats with a single intraperitoneal injection of streptozotocin (60 mg/kg i.p), rats were treated for five, 15 , or 56 days with olive oil ( $1 \mathrm{ml} / \mathrm{kg} \mathrm{p.o})$. Control animals received saline. Blood glucose and body weight were monitored every two weeks. At the end of the treatment, rats were sacrificed and hearts were isolated for mounting on Langedorff's apparatus. The effect of olive oil on oxidative stress and histopathological changes in the cardiac tissues were studied.

\section{Results}

The initial blood glucose and body weight were not significantly different in the control and olive-treated animals. Streptozotocin (60 mg/kg i.p) caused a significant increase in the blood glucose of animals as compared to saline-treated animals. The control, saline-treated diabetic animals exhibited a $100 \%$ incidence of I/R-induced ventricular fibrillation, which was reduced to $0 \%$ with olive oil treatment. The protective effects of olive oil were evident after 15 and 56 days of treatment. Diltiazem, a calcium channel blocker (1 $\mu \mathrm{m} / \mathrm{L}$ ) showed similar results and protected the I/R-induced cardiac disorders. The cardiac tissues isolated from diabetic rats exhibited marked pathological changes in the cardiomyocytes, including decreased glutathione (GSH) and increased oxidative stress (malondialdehyde; MDA). Pretreatment of animals with olive oil (1 ml/kg p.o) increased GSH and decreased MDA levels. Olive oil also improved the diabetic-induced histopathological changes in the cardiomyocytes.

\section{Conclusion}

Olive oil possesses cardiac protective properties against I/R-induced cardiac arrhythmias in rats. It attenuated oxidative stress and diabetes-induced histopathological changes in cardiac tissues. The observed cardiac protectiveness of olive oil in the present investigation may be related to its antioxidant potential.

Categories: Other

Keywords: olive oil, diabetes, ischemia/reperfusion induced cardiac arrhythmias, rat hearts

\section{Introduction}

Olive oil is the primary source of fat in the Mediterranean diet, which is associated with low morbidity and mortality for cardiovascular disease [1]. Olive oil, besides having a high level of monounsaturated fatty acids, contains other components with important biological properties [2]. Diabetes is a major risk for the development of cardiac dysfunctions such as cardiac fibrosis and atrial fibrillation [3-4]. A growing amount of evidence from preclinical and clinical studies has shown that olive oil improves insulin resistance, 
decreases vessel stiffness, and prevents thromboembolism [5-7]. The consumption of the Mediterranean diet, supplemented with olive oil, has been reported with a reduced rate of diabetes and its complications [8]. Extra virgin oil and its fractions possess antioxidant properties and have been shown to exhibit a cardiac protective effect in rat hearts [9]. Several studies have shown that the chronic administration of edible oils, including olive oil, reduced blood pressure, attenuated the loss of hypertension-induced left ventricular cardiomyocytes, and deceased adverse myocardial remodeling in spontaneously hypertensive and streptozotocin (STZ) diabetic rats [10-11]. The preventive role of olive oil against atrial fibrillations has been studied clinically [12]. Other edible oils, such as marine fish oil, prevented ischemia-induced ventricular fibrillation in rats [13]. The significance of olive oil is also well-established in Islamic medicine. Olive oil has a long, traditional use for many health benefits but scientific studies are still scarce to validate its traditional uses. STZ can cause pancreatic $\beta$-cell destruction; it is widely used experimentally as an agent capable of inducing insulin-dependent diabetes mellitus type 1 (T1DM). This model closely resembles human T1DM with chronic pancreatic islet inflammation, insulitis, and insulin deficiency [14]. The STZ-induced diabetic model is widely used to assess the therapeutic potential of new drugs in diabetes-induced complications such as heart problems, hypertension, thromboembolism, endothelial dysfunction, and dyslipidemia [15]. It has been reported that olive consumption decreases the risk of type 2 diabetes and associated complications [16]. Clinical study has revealed that the Mediterranean diet, which comprises olive oil, improves the postprandial glucose and has an antiatherosclerotic effect. Owing to the beneficial cardiovascular effects of edible oils against ischemia-reperfusion-induced cardiac arrhythmias and the diverse medicinal benefits of olive oil in cardiovascular disorders, the aim of our current project was to assess the effects of acute and chronic administration of olive oil against ischemia reperfusion-induced cardiac arrhythmias in isolated diabetic and non-diabetic rat hearts.

\section{Materials And Methods}

\section{Animals}

Male Wistar rats (200-250 g) were obtained from the Animal Care Center, College of Medicine, King Saud University, Riyadh, Saudi Arabia. The experimental protocol was reviewed and approved by the board of Council of Medical Research, College of Medicine, King Saud University, Riyadh, and complied with the National Institutes of Health guidelines for the care and use of laboratory animals. The animals were housed under standard laboratory conditions with a $12 \mathrm{~h}$ light: dark cycle with access to food and water ad libitum.

\section{Chemicals}

Extra virgin olive oil (Hintz foods production, Bremen, Germany) was purchased from the local market. All other chemicals and reagents used in the experiments were of analytical grade.

\section{Induction of diabetes}

Diabetes was induced by the method described elsewhere briefly; male rats (200-250 gm) were treated with freshly prepared streptozotocin (60 mg/kg i.p) [17]. Three days after the administration of streptozotocin injection, fasting glucose levels were measured. Animals with a fasting blood glucose (FBG) level of $>300$ $\mathrm{mg} / \mathrm{dl}$ were included in the study. Rats were divided into different groups: control (saline-treated nondiabetes mellitus, NDM), control (NDM, olive-treated), control (saline-treated diabetes mellitus, DM), and DM treated with olive oil ( $1 \mathrm{ml} / \mathrm{kg}$ orally) for a period of five, 15 , or 56 days. FBG, body weight, and blood pressure were monitored on a weekly basis. After 56 days of treatments with olive oil, the animals were killed and hearts were isolated and assessed for cardiac arrhythmias using the Langendorff apparatus.

\section{Blood pressure measurement in conscious rats}

Arterial blood pressures in the control and olive oil-treated rats were monitored weekly using the tail-cuff method using the MK2000 ST digital blood pressure monitor (Muromachi Kikai Co., Ltd., Japan). Baseline blood pressure was registered before the initiation of the treatment.

\section{Ischemia-reperfusion-induced arrhythmia}

The method described elsewhere was employed [18]. Hearts from male Wistar rats (250-300g, King Saud University breed) were perfused with Krebs-Henseleit solution (composition in $\mathrm{mM}$ : $\mathrm{NaCl} 11$ 8.4, $\mathrm{KCl} 1.3$, $\mathrm{MgSO}_{4} 1.2, \mathrm{KH}_{2} \mathrm{PO}_{4} 1.2, \mathrm{NaHCO}_{3} 25.0, \mathrm{CaCl}_{2} 2.5$, glucose 11.5 ) gassed with $95 \% \mathrm{O}_{2}, 5 \% \mathrm{CO}_{2}$ at a constant flow of $10 \mathrm{ml} / \mathrm{min}$ and maintained at $37^{\circ} \mathrm{C}$. Hearts were isolated from the olive oil-treated rats, a ligature was loosely sited around the left coronary artery at the point where the vessel emerged from under the left atrium.

Isometric contractions, at a resting tension of $2 \mathrm{~g}$, were recorded via a small hook placed in the apex of the left ventricle and attached to a Harvard UFI transducer. Coronary perfusion pressure was monitored with a Washington PT400 pressure transducer. Surface electrical records were obtained from thin wire electrodes placed on the right atrium and apex of the left ventricle. All signals were fed into Harvard transducer interfaces and then into PowerLab/8sp (ADInstruments, Dunedin, New Zealand) connected to a computer. All hearts were allowed a 15 min stabilization period. Following the stabilization period, the coronary artery 
ligature was tightened and released after $10 \mathrm{~min}$. Ventricular arrhythmic activity is recorded for three min post-ligation. Five parameters of ventricular arrhythmias were quantified during the ligation (10 min) and after the release of the ligature for $3 \mathrm{~min}$ : number of premature ventricular contractions (PVCs); percentage incidence of ventricular tachycardia (VT); duration of VT; percentage incidence of ventricular fibrillation (VF); and duration of VF.

\section{Histological and biochemical assays}

At the end of treatment with olive oil or vehicle, the ventricles of the heart were dissected, divided into two portions; one for detecting the underlying structural changes and the other for determining some of the concurrent functional alterations.

Structural Histological Examination

Heart specimens were fixed by $10 \%$ formol saline, processed, embedded, sectioned, and stained by hematoxylin-eosin, Masson's trichrome, or van Gieson stains for the detection of varied structural histopathological changes by light microscope.

Malondialdehyde (MDA) Assay

The principle is based on the reaction of MDA with thiobarbituric acid (TBA) (E. Merck Ltd., Bombay, India), according to the method described elsewhere [19]. The concentration of the MDA-TBA complex was being quantified, measured spectrophotometrically at $532 \mathrm{~nm}$, and expressed as nmol/g wet tissue.

Reduced Glutathione (GSH) Assay

The principle is based on the reaction of reduced GSH with 5.5-dithiobis-(2-nitrobenzoic acid (DTNB) (E. Merck Ltd., Bombay, India), according to the method described elsewhere [20]. The absorbance was measured by the Shimadzu double beam spectrophotometer (UV200S; Shimadzu Corporation, Kyoto, Japan) at $412 \mathrm{~nm}$. The amount of GSH present was calculated using a standard solution of GSH containing $1 \mathrm{mg}$ of $\mathrm{GSH} / \mathrm{ml}$ of 3\% metaphosphoric acid. The increase in the extinction at $412 \mathrm{~nm}$ was proportional to the amount of GSH present and was expressed as nmol/g wet tissue.

\section{Statistical analysis}

All values are represented as mean \pm SEM or percentage incidence. $\mathrm{X} 2$ analysis was used to test the data on the incidence of VT, VF. One-way analysis of variance (ANOVA) followed by the Newman-Keuls test was used for other data. * $\mathrm{p}<0.05, * * \mathrm{p}<0.01$, and ${ }^{* * * *} \mathrm{P}<0.001$ indicated statistical significance. Statistical analysis was performed using Graphpad Prism version 5 (Sydney, Australia).

\section{Results}

\section{Induction of diabetes}

As shown in Figure 1, the control rats treated with normal saline had a fasting blood glucose level of $68 \pm 3$ $\mathrm{mg} / \mathrm{dL}$. Animals that were administered a single i.p injection of streptozotocin $(60 \mathrm{mg} / \mathrm{kg})$ had a fasting blood glucose (FBG) level of $382 \pm 22 \mathrm{mg} / \mathrm{dL}(\mathrm{P}<0.001)$. The mean body weight of the control rats was $288 \pm 6$ $\mathrm{gm}$ while the mean body weight of diabetic rats was $247 \pm 6 \mathrm{gm}$.

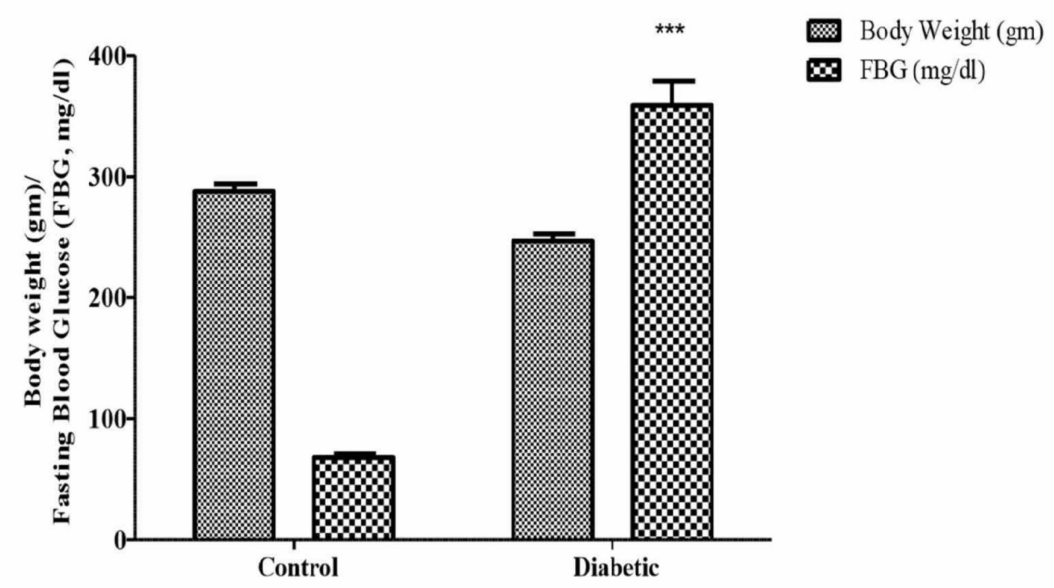




\section{Cureus}

FIGURE 1: Comparison of body weight (g) and fasting blood glucose (FBG; mg/dl) in normal (control) and diabetic rats

Values represent mean \pm SEM $(n=10),{ }^{\star * *} P<0.001$ compared to control.

Animals were treated with a single injection of saline or streptozotocin (60 mg/kg, i.p.).

\section{Effect on mean blood pressure}

The mean blood pressure (MBP) of control rats was $108 \pm 4 \mathrm{mmHg}$ and diabetic rats had an MBP of $115 \pm 5$. Olive oil (1 ml p.o.) for 15 days treatment had no significant effect on blood pressure. The heart rate of diabetic rats was decreased as compared to control animals (Figure 2).

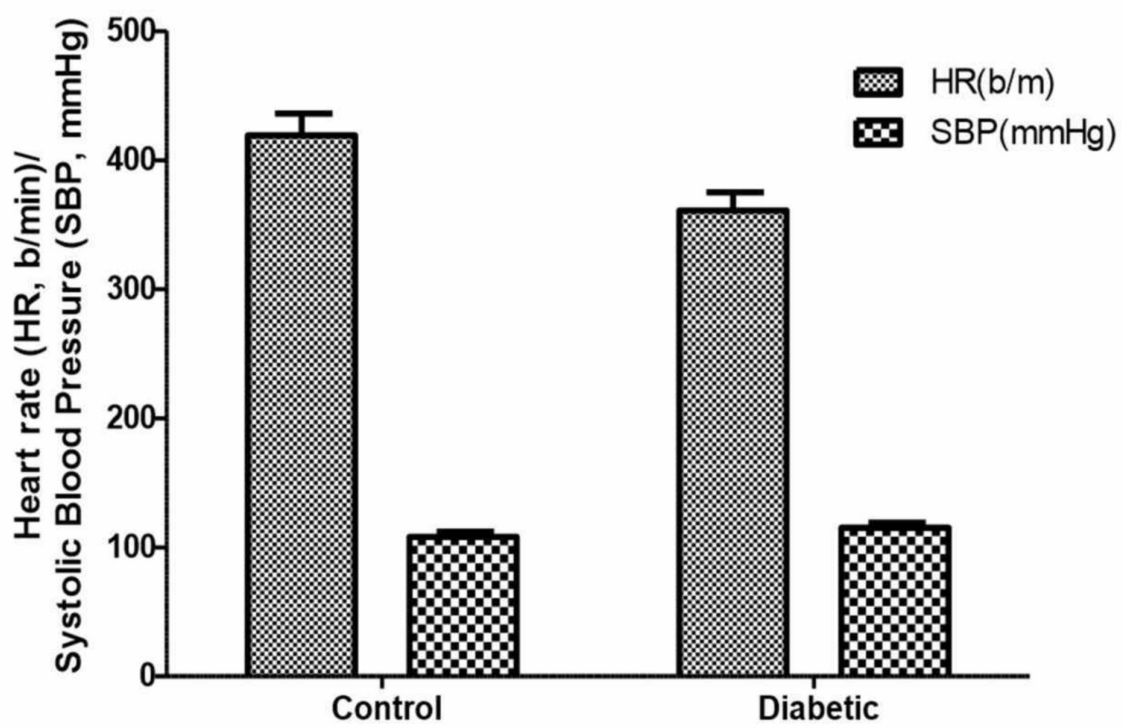

FIGURE 2: Effects of olive oil ( $1 \mathrm{ml} / \mathrm{kg} \mathrm{p.o)}$ on heart rate (HR b/m) and systolic blood pressure (SBP; $\mathrm{mmHg}$ ) in normal (control) and diabetic rats

Values represent mean \pm SEM $(n=10)$

Animals received daily saline or olive ((1 $\mathrm{ml} \mathrm{p.o)} \mathrm{for} 15$ days.

Effect on ischemia-reperfusion induced cardiac arrhythmia (I/R)

As shown in Figures 3-4, in the non-diabetic control rats, the incidence and duration of ventricular fibrillation (VF) were $100 \%$ and $163 \pm 3$ seconds respectively. Pre-treatment of animals with olive oil (1 $\mathrm{ml} / \mathrm{kg}$, p.o.) for five to 56 days significantly decreased the incidence and duration of VF. However, a statistically significant $(\mathrm{P}<0.01)$ decrease in incidence and duration was obtained after 15 and 56 days of treatment with olive oil (Figures 3-4). There was no appreciable difference in the heart rate and perfusion pressure of control and olive oil-treated animals; however, the heart rate of animals treated with olive oil was decreased as compared to saline-treated animals (data not shown). There was a variable effect of olive oil treatment on the premature ventricular counts (PVCs) (Figure 5). 


\section{Cureus}

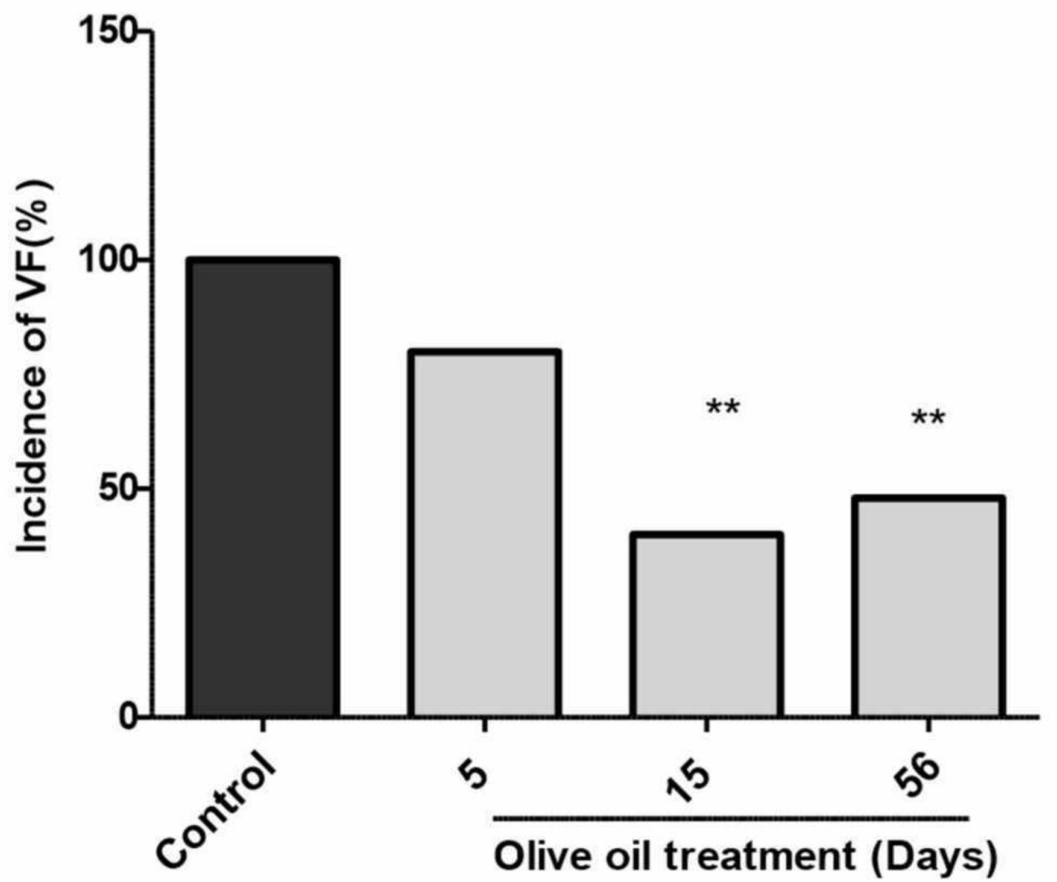

FIGURE 3: Effects of olive oil ( $1 \mathrm{ml} / \mathrm{kg} \mathrm{p.o})$ on the incidence of ischemia/reperfusion-induced ventricular fibrillation (VF) in isolated rat hearts (non-diabetic)

Values represent the percentage of animals showing ventricular fibrillations $(n=10)$. ${ }^{\star \star} P<0.01$ compared to control.

Olive oil (1 $\mathrm{ml} / \mathrm{kg}$ p.o.) was administered for five, 15 , and 56 days.

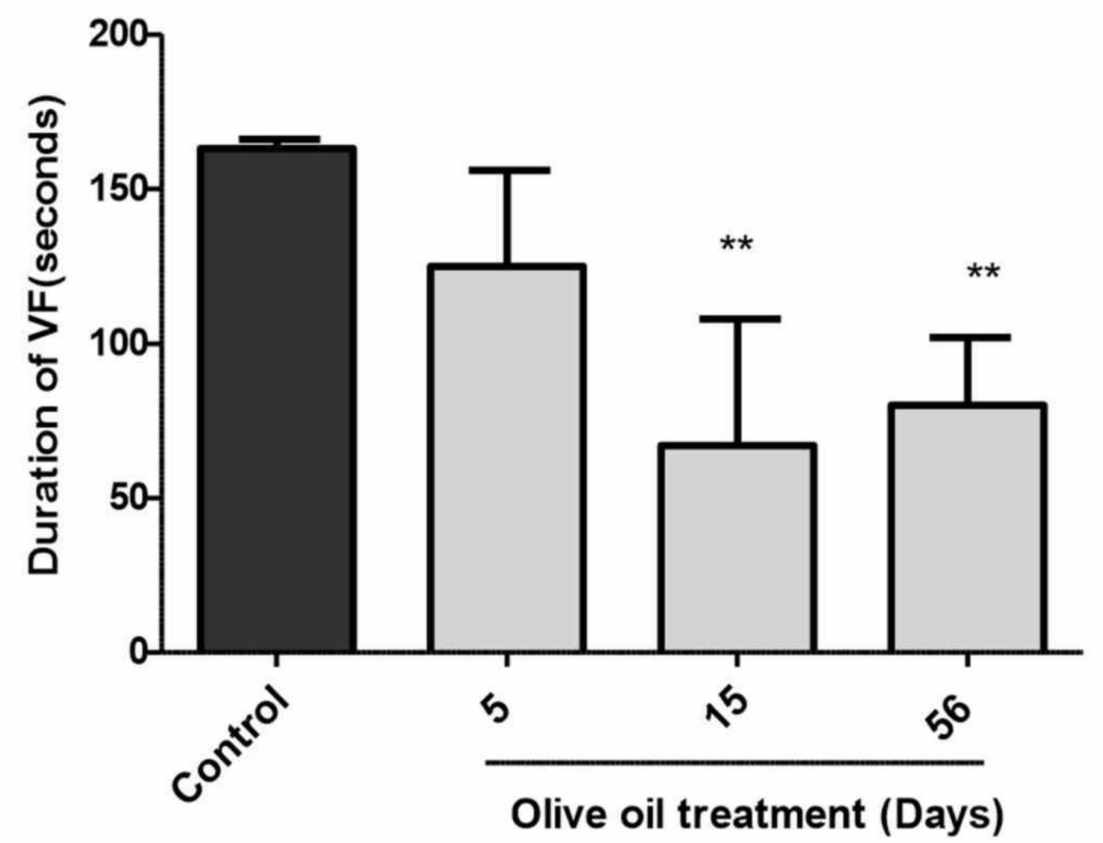




\section{Cureus}

FIGURE 4: Effects of olive oil (1 ml/kg p.o) on the duration of ischemia/reperfusion-induced ventricular fibrillation in isolated rat hearts (non-diabetic)

Values represent mean \pm SEM of $(n=10)$ duration of VF (sec). ${ }^{\star \star} P<0.01$ compared to control.

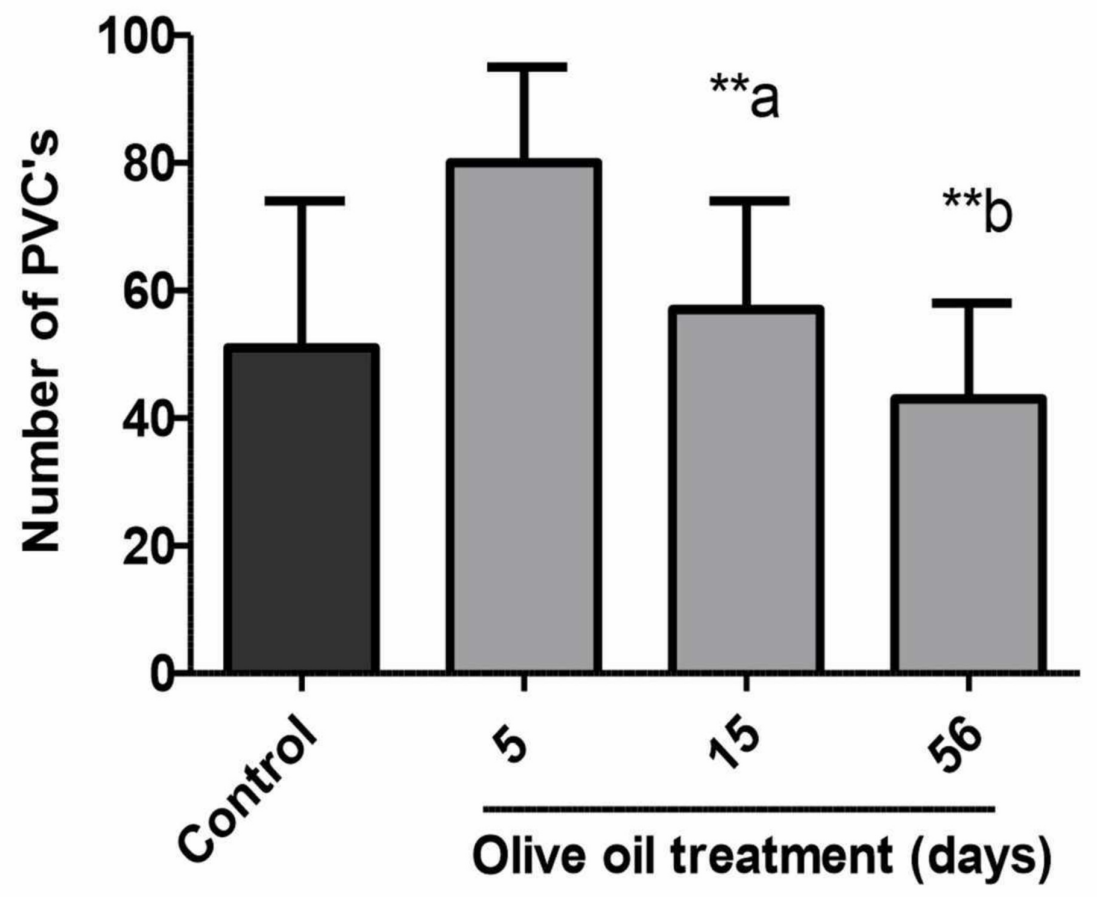

FIGURE 5: Effects of olive oil (1 $\mathrm{ml} \mathrm{p.o)} \mathrm{on} \mathrm{ischemia/reperfusion-}$ induced number of premature ventricular contractions (PVCs) in isolated rat hearts (non-diabetic)

Values represent mean \pm SEM of $(n=10)$ number of PVCs $(n=10) .{ }^{* *} a . P<0.01$ compared to the animal group treated for five days and ${ }^{*} \mathrm{~b}$. $\mathrm{P}<0.01$ compared to control.

Olive oil ( $1 \mathrm{ml} / \mathrm{kg}$ p.o.) was administered orally for five, 15 , or 56 days.

Similar to non-diabetic control animals, the incidence of VF was $100 \%$ in diabetic rats, which was reduced to $0 \%$ with olive oil treatment for 56 days. The incidence of VF was reduced to $70 \%$ in the non-diabetic rats treated with olive oil for 56 days (Figure 6). Similarly, the duration of VF was completely suppressed (Figure 7). Diltiazem ( $1 \mu \mathrm{M} / \mathrm{mL})$, used as the reference drug, produced complete inhibition of both ischemia and reperfusion-induced cardiac arrhythmias (data not shown). 


\section{Cureus}

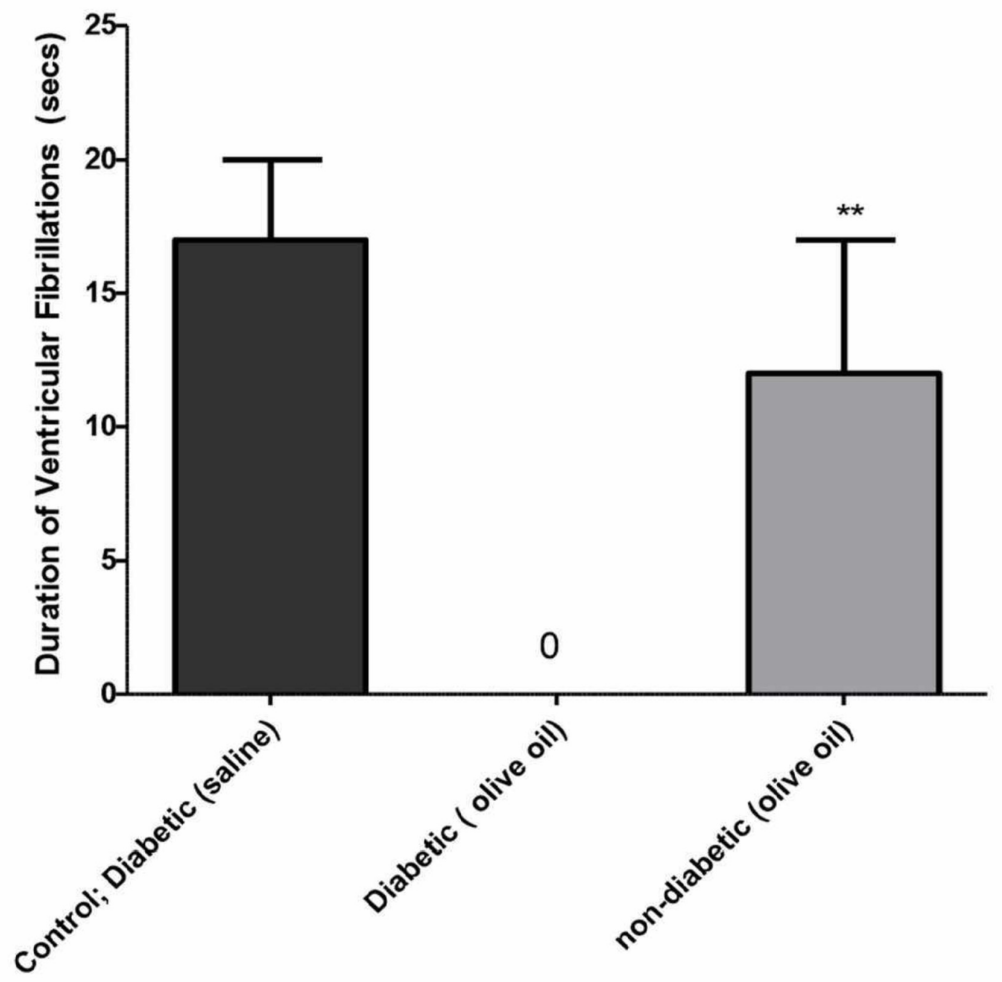

FIGURE 6: Effects of olive oil ( $1 \mathrm{ml} / \mathrm{kg}, \mathrm{p} .0)$ on the duration of ischemia/reperfusion-induced ventricular fibrillation in isolated rat hearts in control (diabetic; saline treated), diabetic (olive oil treated), and non-diabetic (olive oil treated) animals

Olive oil (1 $\mathrm{ml} / \mathrm{kg} \mathrm{p.o)}$ was administered orally for 56 days. None of the diabetic rats treated with olive showed ventricular fibrillation (VF). Values represent the percentage of animals showing ventricular fibrillation $(n=10)$.

** $\mathrm{P}<0.01$ compared to control

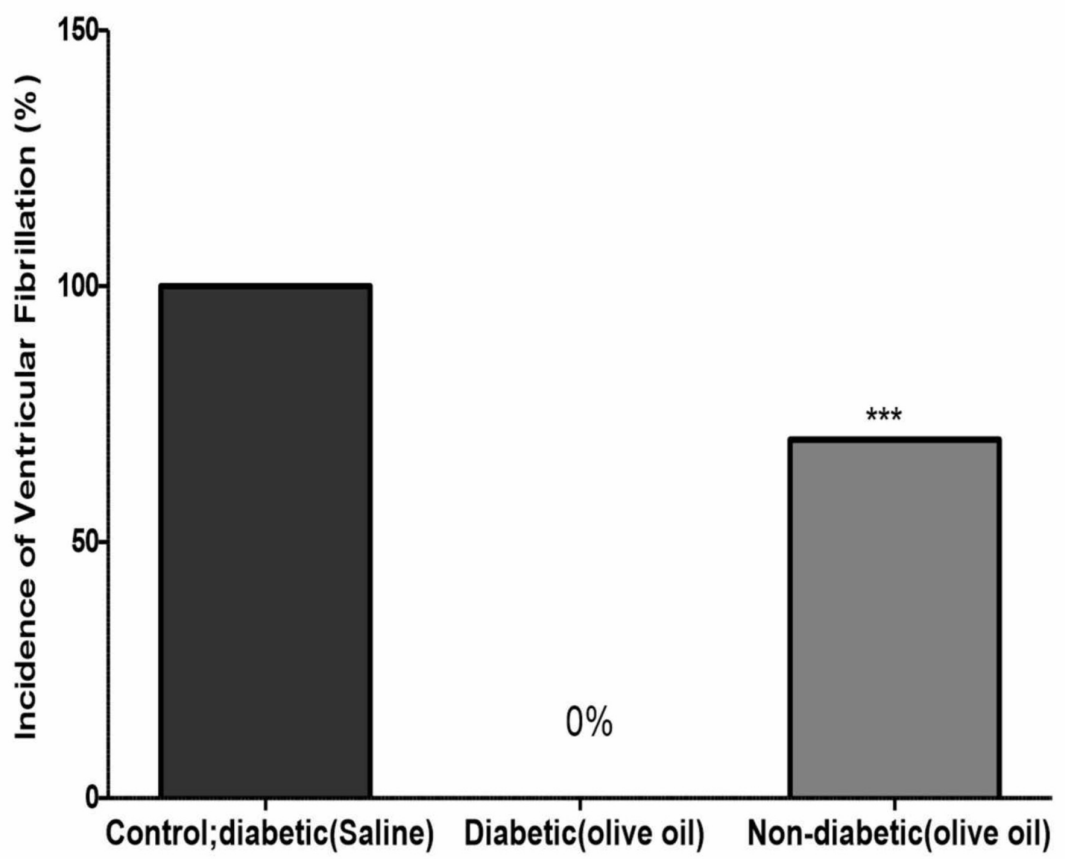




\section{Cureus}

FIGURE 7: Effects of olive oil ( $1 \mathrm{ml} \mathrm{p.o)}$ on the incidence of ischemia/reperfusion-induced ventricular fibrillation in isolated rat hearts in control diabetic (saline), diabetic (olive oil treated), and nondiabetic (olive oil treated)

Values represent the percentage of animals showing ventricular fibrillation (VF) ( $n=10)$. ${ }^{\star \star *} P<0.001$ compared to control.

Olive oil was administered orally for 56 days. None of the diabetic rats treated with olive oil showed VF.

\section{Reduced glutathione (GSH) assays}

As shown in Figure $8 A$, the GSH contents of isolated heart specimens obtained from the non-diabetic control (saline) and control (olive oil) groups were significantly higher ( $\mathrm{P}<0.001)$ compared to the diabetic salinetreated animals (Figure $8 \mathrm{~A}$ ). As shown in the figure, the pretreatment of animals with olive oil $(1 \mathrm{ml} / \mathrm{kg} \mathrm{p.o})$ caused a significant increase in the GSH content of heart specimens obtained from the diabetic olive-treated group $(\mathrm{P}<0.001)$

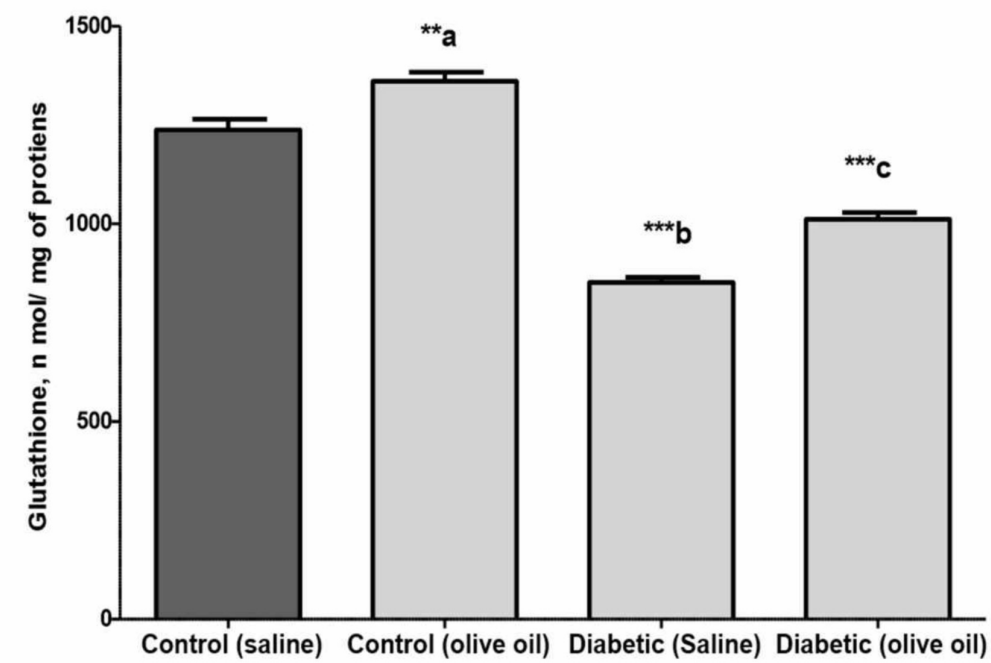

A

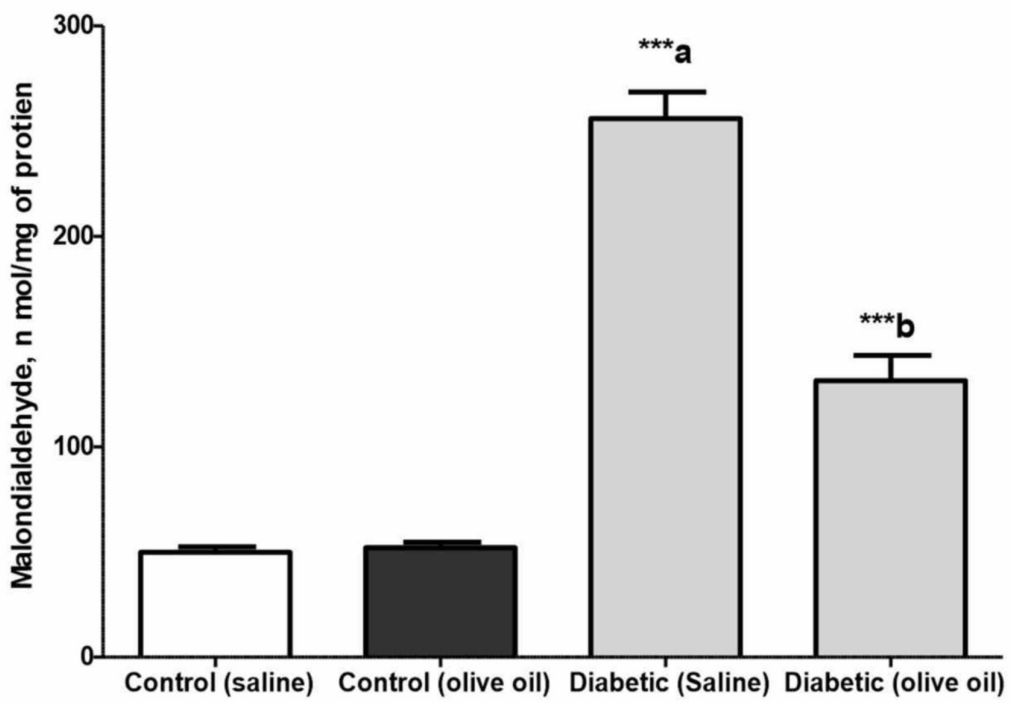




\section{Cureus}

FIGURE 8: Levels of glutathione (A) and malondialdehyde (B) in isolated cardiac tissues of the control and diabetic rats treated with saline or olive oil (1 $\mathrm{ml} / \mathrm{kg}$ p.o.) for 56 days

Data represent mean \pm SEM $(\mathrm{N}=8) .{ }^{* \star} a \mathrm{p}<0.01,{ }^{\star * *} \mathrm{a} \mathrm{P}<0.001$ compared to control saline treated, ${ }^{\star * *} \mathrm{~b} \mathrm{P}<0.001$ compared to diabetic saline treated, and ${ }^{* * *} \mathrm{C}<<0.001$ compared to diabetic saline treated

\section{Malondialdehyde (MDA) assay}

The MDA contents of isolated heart specimens obtained from the control (saline-treated) or control (olive oil-treated) rats were significantly lower $(\mathrm{P}<0.001)$ as compared to the diabetic saline-treated group (Figure $8 B)$. The content of MDA in the diabetic olive oil-treated group was significantly reduced $(\mathrm{P}<0.001)$ as compared to the diabetic saline-treated group. There was no significant difference in the MDA contents of the control saline-treated and control olive oil-treated groups $(\mathrm{P}<0.001)$ (Figure $8 B)$.

\section{Histological analysis}

Figure $9 A$ shows the histopathological changes in the specimens of hearts isolated from normal rats. There was mild interstitial edema in myocardial fibers, with normal myocardial connective tissue distribution (Figure 9A 2\&3). Non-diabetic rats fed on olive oil for 56 days had no changes in the myocardial fibers (Figure $9 B$ 1) and normal collagen distribution (Figure $9 B 2 \& 3$ ):

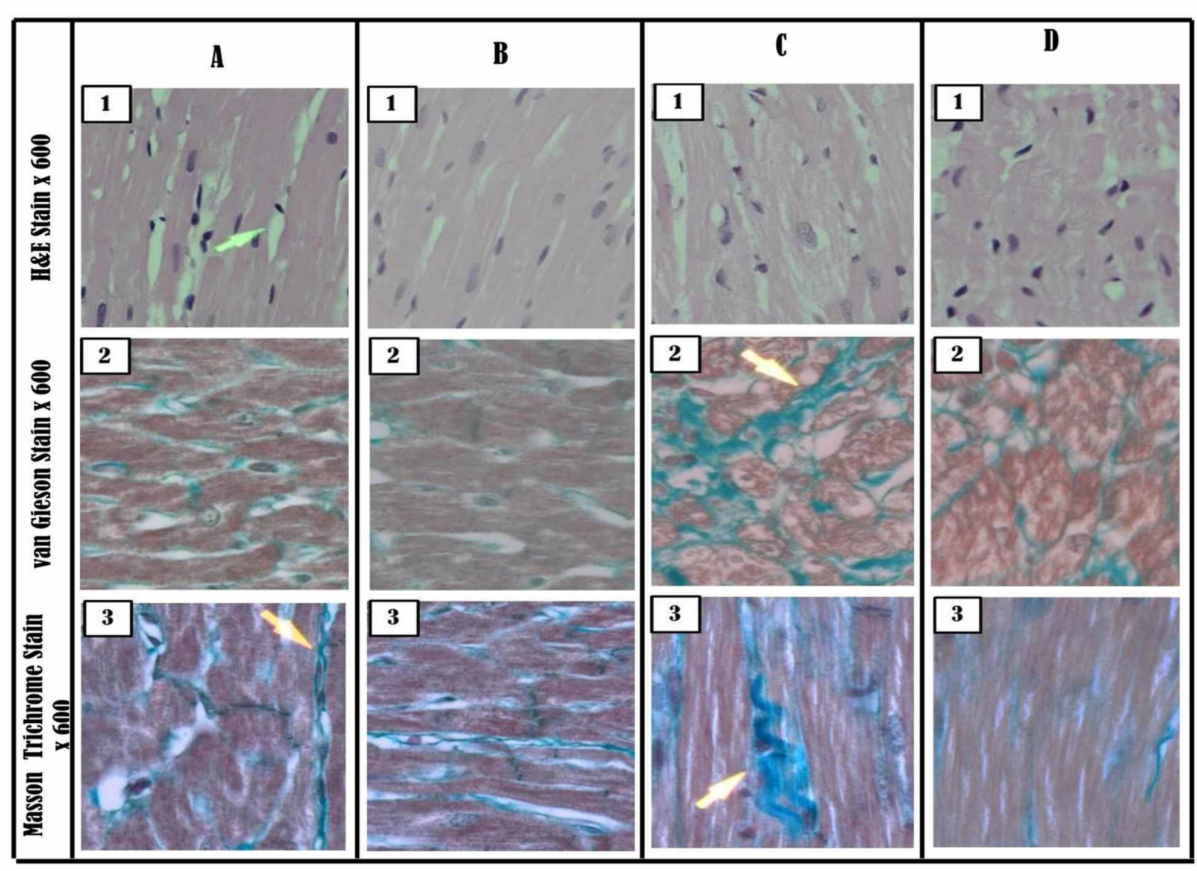

FIGURE 9: Representative images of the histopathological analysis of isolated heart tissues of normal control rats and diabetic rats treated with saline and olive oil

(A) Control saline-treated; A 1: normal myocardial fibers with mild interstitial edema; A 2 \& 3 : normal myocardial connective tissue distribution

(B) Control olive oil-treated; B 1: normal myocardial fibers; B 2 \&3: normal collagen distribution

(C) Diabetic control; C 1: enlarged pleomorphic nuclei with swelling and disruption of myocardial fibers; C 2 \& 3: extensive interstitial fibrosis with bands of dense collagen strands

(D) Diabetic olive oil-treated; D 1: absence of enlarged pleomorphic nuclei, though minimal cellular edema and myocardial fiber disruption is still apparent; D, 2 \& 3: reduction in the amount of interstitial fibrosis

Animals were treated with saline or olive oil $(1 \mathrm{ml} / \mathrm{kg} \mathrm{p.o)}$ for 56 days. 
As shown in Figure $9 \mathrm{C}$ 1, heart tissues from diabetic animals had enlarged pleomorphic nuclei with the swelling and disruption of myocardial fibers. Figure $9 C 2 \& 3$ shows there was extensive interstitial fibrosis with bands of dense collagen strands. On the other hand, diabetic animals treated with olive oil exhibited the absence of enlarged pleomorphic nuclei, though there was minimal cellular edema of cardiomyocytes (Figure 9D 1) with a reduction in the amount of interstitial fibrosis (Figure 9D $2 \& 3$ ).

\section{Discussion}

The Mediterranean diet is associated with low mortality for cardiovascular disease due to its olive oil content $[5,21]$. Olive oil is a rich source of monounsaturated fatty acids and other components with proven beneficial effects on the cardiovascular system [21-22]. In the present study, we assessed the beneficial cardiac effects of olive oil in streptozotocin-induced diabetic rat models. Baseline experiments were conducted in normal rats to establish the dose and duration of olive oil therapy. Animals treated with saline exhibited an increased incidence of cardiac arrhythmias when tested in I/R-induced cardiac arrhythmias in isolated rat hearts. The pretreatment of animals with olive oil for five to 56 days caused a marked reduction in the incidence and duration of VF. The maximum effect was observed on the 56th day of the treatment; therefore, this duration of treatment was chosen for further studies on diabetic rats. Diabetes was induced by a single intraperitoneal injection of streptozotocin. STZ causes pancreatic $\beta$-cell destruction and is a widely used experimental tool to assess the therapeutic potential of new drugs in diabetes-induced complications such as heart problems, hypertension, thromboembolism, endothelial dysfunction, and dyslipidemia [15]. STZ is capable of inducing insulin-dependent diabetes type 1 diabetes mellitus (T1DM), which closely resembles the human T1DM, with chronic pancreatic islet inflammation and insulin deficiency [14]. Diabetic control rats treated with saline had a marked increase in the incidence of ventricular fibrillation. Previous studies have shown that diabetes is a major risk factor of oxidative stress that leads to cardiovascular disorders, including cardiac arrhythmias [4].

Oxidative stress contributes mainly to the pathogenesis of diabetes complications [23]. Normally, free radical superoxide anions generated in the body are rapidly scavenged by natural cellular defense mechanisms, such as superoxide dismutase (SOD) and catalase (CAT), and by the antioxidant action of GSH [24-25]. There is a tremendous increase in oxidative stress and a decrease in the free radical scavenging capability of the body in diabetes mellitus that leads to diabetic complications [26]. The protective effect of olive oil against diabetes-induced cardiac arrhythmia may have occurred by decreasing oxidative stress in the cardiac tissues. This hypothesis was further strengthened when cardiac tissues from olive oil-treated animals exhibited a significant increase in GSH levels and a decrease in MDA levels. MDA is one of the secondary products of lipid peroxidation and is a well-established potential biomarker for oxidative stress [27]. MDA concentration has been an indicator of oxidative imbalance during the onset of several diseases [28]. In the present study, the GSH levels were decreased and the MDA levels were increased in the cardiac tissues isolated from diabetic rats, which was consistent with earlier reports. This, in turn, will lead to increased oxidative stress and cardiac injury in diabetic rats [25]. The decreased level of GSH and increased MDA levels have been reported to be positively correlated with I/R-induced damage [29]. Olive oil is rich in antioxidant components (enzymatic and non-enzymatic) such as catalase, superoxide dismutase, reduced glutathione, ascorbic acid, and reduced nicotinamide adenine dinucleotide phosphate (NADPH)-recycling dehydrogenase [30]. The cardiac protective effect of olive oil may be attributed to these components. Histopathological analysis of cardiac tissues showed that diabetic untreated animals had enlarged pleomorphic nuclei with swelling and disruption of myocardial fibers and there was extensive interstitial fibrosis with bands of dense collagen strands. These histopathological changes were reversed in diabetic animals treated with olive oil, indicating that olive oil prevents diabetes-induced pathological changes in the heart. These findings validate the traditional uses of olive oil in prophetic medicine and the lower incidence of cardiovascular events in people using the Mediterranean diet.

\section{Conclusions}

The present study investigated the effects of olive oil against streptozotocin-induced cardiac disorders using animal models of diabetes and ischemia and reperfusion (I/R)-induced cardiac arrhythmias. Streptozotocininduced diabetes increased the risk of incidence of I/R-induced ventricular fibrillation possibly by increasing oxidative stress in cardiomyocytes. The pretreatment of animals with olive oil prevented these deleterious cardiac changes. The cardiac protective effect of olive oil is most likely mediated via decreasing oxidative stress in the cardiac tissues. These findings validate the traditional use of olive oil for beneficial cardiovascular effects.

\section{Additional Information}

\section{Disclosures}

Human subjects: All authors have confirmed that this study did not involve human participants or tissue. Animal subjects: The experimental protocol was reviewed and approved by the Ethical and Review Board of Medical Research, College of Medicine, King Saud University, Riyadh, and complied with the National Institutes of Health guidelines for the care and use of laboratory animals. Issued protocol number N/A. Conflicts of interest: In compliance with the ICMJE uniform disclosure form, all authors declare the following: Payment/services info: All authors have declared that no financial support was received from 
any organization for the submitted work. Financial relationships: All authors have declared that they have no financial relationships at present or within the previous three years with any organizations that might have an interest in the submitted work. Other relationships: All authors have declared that there are no other relationships or activities that could appear to have influenced the submitted work.

\section{Acknowledgements}

The authors extend their appreciation to the Deanship of Scientific Research at King Saud University, Riyadh, for funding this work through the research group (RG-1439-002).

\section{References}

1. Marcelino G, Hiane PA, Freitas KC, Santana LF, Pott A, Donadon JR, Guimarães RCA: Effects of olive oil and its minor components on cardiovascular diseases, inflammation, and gut microbiota. Nutrients. 2019, 11:E1826. 10.3390/nu11081826

2. Chrysohoou C, Pitsavos C, Lazaros G, Skoumas J, Tousoulis D, Stefanadis C: Determinants of all-cause mortality and incidence of cardiovascular disease (2009 to 2013) in older adults: the Ikaria Study of the Blue Zones. Angiology. 2015, 67:541-548. 10.1177/0003319715603185

3. Bugger H, Bode C: The vulnerable myocardium. Diabetic cardiomyopathy. Hamostaseologie. 2015, 35:17-24. 10.5482/hamo-14-09-0038

4. Saito S, Teshima Y, Fukui A, et al.: Glucose fluctuations increase the incidence of atrial fibrillation in diabetic rats. Cardiovasc Res. 2014, 104:5-14. 10.1093/cvr/cvu176

5. Covas MI: Olive oil and the cardiovascular system. Pharmacol Res. 2007, 55:175-186. 10.1016/j.phrs.2007.01.010

6. Visioli F, Caruso D, Grande S, et al.: Virgin Olive Oil Study (VOLOS): vasoprotective potential of extra virgin olive oil in mildly dyslipidemic patients. Eur J Nutr. 2005, 44:121-127. 10.1007/s00394-004-0504-0

7. Huang CL, Sumpio BE: Olive oil, the Mediterranean diet, and cardiovascular health . J Am Coll Surg. 2008, 207:407-16. 10.1016/j.jamcollsurg.2008.02.018

8. Ceriello A, Esposito K, La Sala L, et al.: The protective effect of the Mediterranean diet on endothelial resistance to GLP-1 in type 2 diabetes: a preliminary report. Cardiovasc Diabetol. 2014, 13:140. 10.1186/s12933-014-0140-9

9. Ghorbel I, Khemakhem M, Boudawara O, et al.: Effects of dietary extra virgin olive oil and its fractions on antioxidant status and DNA damage in the heart of rats co-exposed to aluminum and acrylamide. Food Funct. 2015, 6:3098-3108. 10.1039/c5fo00342c

10. Aguila MB, Pinheiro AR, Mandarim-de-Lacerda CA: Spontaneously hypertensive rats left ventricular cardiomyocyte loss attenuation through different edible oils long-term intake. Int J Cardiol. 2005, 100:461466. 10.1016/i.ijcard.2004.12.008

11. Medeiros FJ, Mothe CG, Aguila MB, Mandarim-de-Lacerda CA: Long-term intake of edible oils benefits blood pressure and myocardial structure in spontaneously hypertensive rat (SHR) and streptozotocin diabetic SHR. Prostaglandins Other Lipid Mediat. 2005, 78:231-248. 10.1016/j.prostaglandins.2005.09.001

12. Martinez-Gonzalez MA, Toledo E, Aros F, et al.: Extravirgin olive oil consumption reduces risk of atrial fibrillation: the PREDIMED (Prevencion con Dieta Mediterranea) trial. Circulation. 2014, 130:18-26. 10.1161/circulationaha.113.006921

13. Nair SS, Leitch JW, Falconer J, Garg ML: Prevention of cardiac arrhythmia by dietary (n-3) polyunsaturated fatty acids and their mechanism of action. J Nutr. 1997, 127:383-393. 10.1093/jn/127.3.383

14. Mabley JG, Rabinovitch A, Suarez-Pinzon W, et al.: Inosine protects against the development of diabetes in multiple-low-dose streptozotocin and nonobese diabetic mouse models of type 1 diabetes. Mol Med. 2003, 9:96-104. 10.2119/2003-00016.Mabley

15. Tan Z, Xu Z, Gui Q, Wu W, Yang Y: Gliquidone versus metformin: differential effects on aorta in streptozotocin induced diabetic rats. Chin Med J (Engl). 2014, 127:1298-1303.

16. Guasch-Ferre M, Hruby A, Salas-Salvado J, Martinez-Gonzalez MA, Sun Q, Willett WC, Hu FB: Olive oil consumption and risk of type 2 diabetes in US women. Am J Clin Nutr. 2015, 102:479-486. 10.3945/ajcn.115.112029

17. Mushtaq N, Schmatz R, Ahmed M, et al.: Protective effect of rosmarinic acid against oxidative stress biomarkers in liver and kidney of strepotozotocin-induced diabetic rats. J Physiol Biochem. 2015, 71:743751. 10.1007/s13105-015-0438-4

18. Bukhari IA, Almotrefi AA, Mohamed OY, Al-Masri AA, Sheikh SA: Protective effect of fenofibrate against ischemia-/reperfusion-induced cardiac arrhythmias in isolated rat hearts. Fundam Clin Pharmacol. 2018, 32:141-146. 10.1111/fcp.12342

19. Buege JA, Aust SD: Microsomal lipid peroxidation. Methods Enzymol. 1978, 52:302-310. 10.1016/s00766879(78)52032-6

20. Jollow DJ, Mitchell JR, Zampaglione N, Gillette JR: Bromobenzene-induced liver necrosis. Protective role of glutathione and evidence for 3,4-bromobenzene oxide as the hepatotoxic metabolite. Pharmacology. 1974, 11:151-169. 10.1159/000136485

21. Covas MI, de la Torre R, Fito M: Virgin olive oil: a key food for cardiovascular risk protection . Br J Nutr. 2015, 113:19-28. 10.1017/s0007114515000136

22. Bukhari IA, Sheikh BY, Almotrefi AA, Yousaf O, Mahmood A: Cardiac protective effect of olive oil against ischemia reperfusion-induced cardiac arrhythmias in isolated diabetic rat hearts. Int J Pharmacol Pharm Sci. 2015, 9:1.

23. Kato T, Yamashita T, Sekiguchi A, et al.: What are arrhythmogenic substrates in diabetic rat atria? . J Cardiovasc Electrophysiol. 2006, 17:890-894. 10.1111/j.1540-8167.2006.00528.x

24. Giacco F, Brownlee M, Schmidt AM: Oxidative stress and diabetic complications. Circ Res. 2010, 107:10581070. 10.1161/circresaha. 110.223545 


\section{Cureus}

25. Moskaug JO, Carlsen H, Myhrstad MC, Blomhoff R: Polyphenols and glutathione synthesis regulation. Am J Clin Nutr. 2005, 81:277-283. 10.1093/ajcn/81.1.277S

26. Rains JL, Jain SK: Oxidative stress, insulin signaling, and diabetes. Free Radic Biol Med. 2011, 50:567-575. 10.1016/j.freeradbiomed.2010.12.006

27. Nielsen F, Mikkelsen BB, Nielsen JB, Andersen HR, Grandjean P: Plasma malondialdehyde as biomarker for oxidative stress: reference interval and effects of life-style factors. Clin Chem. 1997, 43:1209-1214. 10.1093/clinchem/43.7.1209

28. Sheweita SA, Sheikh BY: Can dietary antioxidants reduce the incidence of brain tumors? . Curr Drug Metab. 2011, 12:587-593. 10.2174/138920011795713733

29. Chen L, Qi Y, Yang X: Neuroprotective effects of crocin against oxidative stress induced by ischemia/reperfusion injury in rat retina. Ophthalmic Res. 2015, 54:157-168. 10.1159/000439026

30. Lopez-Huertas E, Del Rio LA: P53 - characterization of antioxidant enzymes and peroxisomes of olive (Olea europaea L.) fruits. Free Radic Biol Med. 2014, 75:S39. 10.1016/j.freeradbiomed.2014.10.785 